\section{PTH-27 RISK-STRATIFIED FIT FOR URGENT COLONOSCOPY IN LYNCH SYNDROME: A CLINICAL SERVICE THROUGHOUT THE COVID-19 PANDEMIC}

${ }^{1}$ Anne Lincoln*, ${ }^{2}$ Anne Lincoln, ${ }^{3}$ Sally Benton, ${ }^{2}$ Peter Sasieni. ${ }^{1}$ North West London Hospitals NHS Trust, St. Mark's Hospital, Harrow, Middlesex, UK; ${ }^{2}$ King's College London, London, UK; ${ }^{3}$ NHS Bowel Cancer Screening South of England Hub, Guildford, UK

\subsection{6/gutjnl-2021-BSG.342}

Introduction Lynch syndrome (LS) is an autosomal dominant inherited disorder characterised by pathogenic variants within the mismatch repair (MMR) genes, MLH1, MSH2, MSH6, $P M S 2$, and a variant in EPCAM, which regulates MSH2 expression and results in an increased risk of several cancers, particularly colorectal cancer with an annual incidence rate of 3-5\%. The faecal immunochemical test (FIT) is currently used in nonLS symptomatic and screening populations to guide subsequent colonoscopy. We report preliminary results from an NHS England endorsed clinical service implemented during the COVID19 pandemic (June-December 2020) which used FIT to prioritise colonoscopy in the highest risk LS patients in response to limited colonoscopy capacity throughout the pandemic.

Methods Regional genetic and endoscopy services across England were invited to participate, with the support of the British Society of Gastroenterology (BSG). Patient eligibility was determined by 1) Diagnosis of Lynch Syndrome 2) Planned colonoscopic surveillance between 1 March 2020 and 31 December 2020. Requests for FIT testing from participating NHS Trusts were sent to the NHS Bowel Cancer Screening South of England Hub in Guildford. The Hub sent patients a FIT kit (OC-Sensor ${ }^{\mathrm{TM}}$ (Eiken, Japan)), instructions for use, a questionnaire, and a pre-paid return envelope. Laboratory reports with faecal haemoglobin (f-Hb) results were returned electronically for clinical action. LS patients were risk-stratified for colonoscopy based upon the following $\mathrm{f}-\mathrm{Hb}$ thresholds: (1) $\mathrm{f}-\mathrm{Hb} \geq 10 \mu \mathrm{g} \mathrm{Hb} / \mathrm{g}$ faeces: triaged for colonoscopy via the two-week wait (2WW) pathway, (2) $\mathrm{f}-\mathrm{Hb} \leq 10 \mu \mathrm{g} \mathrm{Hb} / \mathrm{g}$ faeces: schedule patients for colonoscopy within 6-12 weeks, where local endoscopy service availability permits.

Results Fourteen centres across England participated in the clinical service evaluation from 9th June 2020 to 1st December 2020. An uptake rate of $63 \%$ was observed from this cohort (269/428 invites). Of the 269 patients who returned their FIT devices, 20\% ( $\mathrm{n}=55)$ had $\mathrm{f}-\mathrm{Hb} \geq 10 \mu \mathrm{g} \mathrm{Hb} / \mathrm{g}$ faeces and met criteria for urgent colonoscopy triage via the $2 \mathrm{WW}$ pathway. $29 \%(n=77)$ of patients had a $\mathrm{f}-\mathrm{Hb}$ that was greater than the limit of detection of the assay $(6 \mu \mathrm{g} \mathrm{Hb} / \mathrm{g}$ faeces) yet below $10 \mu \mathrm{g} \mathrm{Hb} / \mathrm{g}$ faeces. 22 patients $(8 \%)$ had $\mathrm{f}-\mathrm{Hb}$ between 6 and $9.9 \mu \mathrm{g} \mathrm{Hb} / \mathrm{g}$ faeces. In a subgroup analysis of 67 patients from St Mark's Hospital, the adenoma detection rate was $42.4 \%$, and $9 / 67$ proceeded to priority colonoscopy based on their FIT findings.

Conclusions Implementation of this clinical service during the COVID-19 pandemic has demonstrated clinical value for LS patients requiring routine surveillance. Further investigation on the efficacy of this novel intervention is warranted, namely as it pertains to additional data analysis to include endoscopy findings for participating LS patients to discern FIT sensitivity and specificity, which will be further explored under the auspices of a multi-centre prospective research study which is presently in development.

\section{PTH-28 ELEMENTAL FORMULA AND LOFFLEX NUTRITION THERAPY; AN EFFECTIVE BUT NEGLECTED TREATMENT STRATEGY IN CROHN'S DISEASE}

${ }^{1,2}$ Nuur Hj Zulkiflee*, ${ }^{3,4}$ Carlos Montoya, ${ }^{2}$ Adele Rostami, ${ }^{2}$ James Irwin, ${ }^{2}$ Kamran Rostami. ${ }^{1}$ University Of Otago, Wellington, New Zealand; ${ }^{2}$ Department of Gastroenterology and Nutrition MidCentral DHB Palmerston North Hospital, Palmerston North, New Zealand; ${ }^{3}$ AgResearch, Palmerston North, New Zealand; ${ }^{4}$ Massey University, Palmerston North, New Zealand

\subsection{6/gutjnl-2021-BSG.343}

Introduction Nutrition therapy in treatment of Chron's Disease (CD) is not adequately highlighted in adult guidelines despite strong evidence supporting its therapeutic efficacy and thus, this option is often not offered to Crohn's patients during gastroenterology consultations. In this retrospective study, we aim to evaluate the effectiveness of nutrition therapy using exclusive Elemental Formula for 2 weeks followed by a transition to Low Fat/Fiber Limited Exclusion (LOFFLEX) diet for at least 3-4 weeks in treating adults with active CD.

Methods 38 patients with active CD were recruited from Palmerston North Hospital. Only 28 fulfilled the inclusion criteria. Participants were treated with exclusive Elemental formula for 2 weeks with a transition to a LOFFLEX diet for 3-4 weeks and finally reintroduction to normal diet.

The primary outcomes measured were disease activity defined by the Harvey Bradshaw Index (HBI), prevalence of diarrhoea, abdominal pain, blood in stools, general well-being and changes in inflammatory markers of C-reactive protein (CRP) and white blood cell count (WBC). Statistical analyses were performed using SAS (SAS/STAT version 9.4; SAS Institute Inc., Cary, NC, USA).

Results Following exclusive Elemental formula and/or LOFFLEX nutrition therapy, full clinical remission was achieved in 22 patients. This was reflected by substantial improvements in disease activity and inflammatory markers across all patients at the end of the study. Mean HBI score fell from $7.85 \pm 0.63$ at baseline to $3.42 \pm 1.19$ (a $56.4 \%$ improvement; $\mathrm{P}<0.001$ ). Frequency of diarrhea and abdominal pain was halved from 0.81 \pm 0.08 to $0.27 \pm 0.17 \quad(\mathrm{P}<0.01)$ and $0.35 \pm 0.18 \quad(\mathrm{P}<0.05)$ respectively. Frequency of blood in stools decreased by $13 \%$ $(\mathrm{P}<0.05)$ and $\mathrm{CRP}$ levels improved by $67.8 \%(\mathrm{P}<0.05)$ from baseline.

Conclusion Nutrition therapy with exclusive Elemental formula followed by LOFFLEX diet was highly successful and effective in inducing full clinical remission in $78.5 \%$ of cases and the

Abstract PTH-28 Table 1 Effect of nutrition therapy with Elemental formula and LOFFLEX diet on disease activity, symptom prevalence and inflammatory markers (Mean \pm SEM)

\begin{tabular}{lll}
\hline Parameters & Start & End \\
\hline Harvey Bradshaw Index & $7.85 \pm 0.63$ & $3.42 \pm 1.19^{* * *}$ \\
Diarrhoea Prevalence & $0.81 \pm 0.08$ & $0.27 \pm 0.17^{* *}$ \\
Abdominal Pain Prevalence & $0.81 \pm 0.08$ & $0.35 \pm 0.18^{*}$ \\
Blood in stools Prevalence & $0.49 \pm 0.09$ & $0.14 \pm 0.09^{*}$ \\
CRP & $1.99 \pm 0.26$ & $0.64 \pm 0.51^{*}$ \\
WBC & $8.84 \pm 0.58$ & $8.37 \pm 0.91$ \\
\hline
\end{tabular}

${ }^{*} \mathrm{P}<0.05 ;{ }^{* *} \mathrm{P}<0.01 ;{ }^{* *} \mathrm{P}<0.001$ differ from the normal diet 
remaining $21.5 \%$ reported improvement in severity of disease. Thus, we strongly advocate that nutrition therapy should at least be offered as a treatment modality to every Crohn's patient during gastroenterology consultations.

\section{PTH-29 INTENTIONAL FOREIGN BODY INGESTION DURING THE COVID-19 CRISIS: A CRY FOR HELP?}

Luke Hanna*, Nina-Joyce Shehata, Tracie Jarvis, Fergus Thursby-Pelham, Sharmila Subramaniam. Department of Gastroenterology, Portsmouth Hospitals University NHS Trust, UK

\subsection{6/gutjpl-2021-BSG.344}

Introduction Endoscopy was significantly affected by the COVID-19 pandemic in the past year, meaning any increased demand placed significant strains on services. Rising rates of intentional foreign body ingestion (IFBI) requiring urgent endoscopy impacted on our centre in this period leading us to compare rates with pre-pandemic levels and analyse it against key dates in the UK coronavirus timeline.

Methods Endoscopic records in a large acute NHS trust in South England were examined retrospectively between 01/01/ 2019 - 31/12/2020 to identify gastroscopies for foreign body removal. Procedures were reviewed and individuals' records interrogated to identify information on their backgrounds and prior behaviours. Rates of IFBI were examined against contemporary events of the COVID pandemic.

Results 122 endoscopies were identified for foreign body removal. Of these: $46(37.7 \%)$ were for IFBI, 69 (56.6\%) for food bolus removal, and 7 (5.7\%) for accidental foreign body ingestion. Whilst rates for food bolus dropped by almost a third in 2020 (compared to 2019), we noted an 8.2 times increase in IFBI procedures for the same period. 8 individual patients contributed to the IFBI workload. $54.3 \%$ of IFBI endoscopies required general anaesthetic and 95\% involved sharp or dangerous objects.

Rates were analysed against the COVID timeline (Table 1). Between 01/01/2020 - 23/03/2020, when global COVID alerts were rising, levels of IFBI were relatively high with 5 procedures performed (equal to total 2019 number). From 23/03/ 2020-10/05/2020, when the UK was in its 1st lockdown, numbers dropped with only one case recorded. After this point levels rose dramatically with 1.13 procedures a week logged until 31/10/2020, when the UK re-entered lockdown. The 2nd lockdown, and later tiered system, did not see dramatic reductions in activity as cases continued at 0.80 week until the end of 2020 .

Conclusions There has been a large increase in IFBI requiring emergency endoscopy in 2020. Mental health stresses during the pandemic seem to have had a role in susceptible individuals reaching crises point in this behaviour. Sustained effects of increased anxiety, in combination with patchy access to mental health services, may have led to this manifestation of selfharm. This represents yet another unforeseen consequence of the COVID pandemic that has affected the utilisation of endoscopy services locally.

\section{PTH-30 THE FIRST UK DIETITIANS PROVIDING ADVANCED PRACTITIONER GI SERVICES}

Anupam Rej* ${ }^{*}$ Rachel Buckle, Anupam Rej, Kumar Basu, Neil Thompson, Nicola Moran, Nick Trott, Imran Aziz, David Sanders. Academic Unit of Gastroenterology, Sheffield Teaching Hospital NHS Foundation Trust, Sheffield, UK

\subsection{6/gutjnl-2021-BSG.345}

Introduction Clinical demand for gastrointestinal (GI) services in the United Kingdom (UK) has grown exponentially and the mean annual consultant expansion of $5 \%$ over the last 10 years has still not dealt with the unmet need. ${ }^{1}$ As a result $43 \%$ of new consultant appointments are vacant and private contractors are filling this space. ${ }^{1}$ We provide data on the first UK GI dietitians to deliver an advanced practitioner role using a payment by results model $(\mathrm{PbR})$.

Methods Two Band 6 dietitians were trained by a physician over a 12-month period (cost $£ 40,500$ per dietitian per annum) to undertake outpatient medical gastroenterology clinics. In addition, they were enrolled into a MSc in advanced clinical practice (cost $£ 8490$ per dietitian). Outpatient clinics assessing patients with symptoms compatible with irritable bowel syndrome (IBS) were performed, initially physician-led, followed by dietetic-led. Patients were invited to assess their clinic satisfaction on a scale from 0-10. Clinical notes were reviewed subsequently to see the diagnostic outcomes of these patients. Cost analysis of income generation from clinics was also performed.

Results 91 clinical notes were reviewed (mean age 38 years, $66 \%$ female $[n=60])$, with $73 \%(n=66)$ having a diagnosis of IBS, $12 \%[\mathrm{n}=11]$ bile acid diarrhoea, 4\% [n=4] functional diarrhoea, 2\% [n=2] microscopic colitis and 9\% $[n=8]$ having another diagnosis. 62\% $[\mathrm{n}=56]$ had been seen by a physician, with $38 \%[n=35]$ being seen by a dietitian. There was no statistically significant difference in clinic satisfaction between physician and dietetic-led clinics (physician-led mean 9.2 \pm 1.5 vs dietitian-led mean $9.4 \pm 1.1, p=0.50)$. Annual income generation was calculated on the basis of 84 clinics annually per dietitian, with 3 new patients and 7 follow ups (total income generation of $£ 68.2 \mathrm{k}$ [ $£ 35.3 \mathrm{k}$ new patients, $£ 32.9 \mathrm{k}$ follow ups]). The cost to employ a band 7 dietitian to deliver this service is $£ 53.5 \mathrm{k}$ (midpoint of scale), providing an estimated net surplus of $£ 14.7 \mathrm{k}$ based on the $2020 / 21 \mathrm{PbR}$ tariff to the directorate.

Conclusions Dietetic-led outpatient clinics for IBS patients can lead to an annual surplus of $£ 14.7 \mathrm{k}$ per dietitian, with no difference in patient satisfaction between physician and dietitian-

Abstract PTH-29 Table 1 IFBI endoscopies against COVID timeline

\begin{tabular}{|c|c|c|c|c|c|c|}
\hline \multirow[t]{2}{*}{ Endoscopies for IFBI } & \multirow[t]{2}{*}{ Total year 2019} & \multicolumn{4}{|l|}{ Jan-Dec 2020} & \multirow[t]{2}{*}{ Total year 2020} \\
\hline & & $\begin{array}{l}01 / 01 / 20-23 / 03 / 20 \\
\text { (pre-lockdown) }\end{array}$ & $\begin{array}{l}23 / 03 / 20-10 / 05 / 20 \\
\text { (1st lockdown) }\end{array}$ & $\begin{array}{l}10 / 05 / 20-31 / 10 / 20 \\
\text { (variable easing of restrictions) }\end{array}$ & $\begin{array}{l}31 / 10 / 20-31 / 12 / 20 \\
\text { (2nd national lockdown }+ \text { tiered restrictions) }\end{array}$ & \\
\hline Total number & 5 & 5 & 1 & 28 & 7 & 41 \\
\hline Procedures per week & 0.10 & 0.43 & 0.15 & 1.13 & 0.80 & 0.79 \\
\hline
\end{tabular}

